\title{
OPTIMIZACIÓN DE LA PRODUCCIÓN DE FRUCTOOLIGOSACÁRIDOS A PARTIR DEL EXTRACTO DE ALGARROBO UTILIZANDO FRUCTOSILTRANSFERASAS
}

\author{
Carmen Peña-Suasnabar*, Amparo Iris Zavaleta
}

\begin{abstract}
RESUMEN
Los fructooligosacáridos (FOS) son polímeros de fructosa, unidos mediante enlaces glucosídicos $\beta(2-1)$, considerados edulcorantes de bajo aporte calórico con función prebiótica que pueden ser obtenidos a partir de sacarosa por biosíntesis. Los frutos del algarrobo (Prosopis pallida) contienen principalmente carbohidratos y, en particular, alta concentración de sacarosa. Por tal motivo, se optimizó la producción de FOS a partir del extracto de algarrobo usando pectinasa de A. aculeatus. La metodología de superficie de respuesta se aplicó para optimizar los parámetros que afectan la producción como: tiempo, temperatura y extracto de algarrobo; después se verificaron experimentalmente. El proceso de biosíntesis de FOS se realizó con extracto de algarrobo a las concentraciones de 20, 35 y $50{ }^{\circ} \mathrm{Bx}$, pectinasa a 10,35 y $61 \mathrm{mg}$, a 45,55 y $65^{\circ} \mathrm{C}$ durante 0,$5 ; 9,5$ y $18 \mathrm{~h}$, con agitación a $150 \mathrm{rpm}$. Los métodos de análisis para glucosa y FOS fueron glucosa oxidasa y AOAC 999,03 respectivamente y el programa Minitab 16. Las condiciones óptimas de reacción para la producción de FOS fueron: extracto de algarrobo $38^{\circ} \mathrm{Bx}$, pectinasa $31 \mathrm{mg}, 54{ }^{\circ} \mathrm{C}$, $150 \mathrm{rpm}$ y $12 \mathrm{~h}$. El rendimiento experimental en el extracto transformado fue FOS 16,1\% cuantificados por HPLC.
\end{abstract}

Palabras clave: fructooligosacáridos, FOS, algarrobo, Prosopis pallida, pectinasa

\section{OPTIMIZATION OF A FRUCTOOLIGOSACCHARIDES PRODUCTIVE PROCESS FROM CAROB EXTRACT USING FRUCTOSILTRANSFERASES}

\begin{abstract}
SUMMARY
Fructooligosaccharides (FOS) are fructose polymers, linked by $\beta$-glucosidic bonds (2-1), considered low caloric food sweeteners with prebiotic function and can be obtained from sucrose by biosynthesis. The fruits of the carob tree (Prosopis pallida) contain mainly carbohydrates where sucrose stands out. For this reason, the production of FOS from carob

Laboratorio de Biología Molecular, Facultad de Farmacia y Bioquímica, Universidad Nacional Mayor de San Marcos, Lima 1, Perú

Carmen.pena@unmsm.edu.pe
\end{abstract}


extract was optimized using a pectinase from $A$. aculeatus. The response surface methodology using was applied to optimize the parameters that affected production: time, temperature and carob extract; then they were verified experimentally. The biosynthesis process of FOS was carried out with carob extract at the concentrations of 20,35 and $50^{\circ} \mathrm{Bx}$, Pectinase at 10 , 35 and $61 \mathrm{mg}$, at 45,55 and $65^{\circ} \mathrm{C}$ for $0,5,9,5$ and $18 \mathrm{~h}$, with stirring at $150 \mathrm{rpm}$ The analysis methods were glucose oxidase and AOAC 999.03, for glucose and FOS respectively and the Minitab 16 program. The optimal reaction conditions for the production of FOS were: carob extract $38^{\circ} \mathrm{Bx}$, enzyme $31 \mathrm{mg}, 54{ }^{\circ} \mathrm{C}, 150 \mathrm{rpm}$ and $12 \mathrm{~h}$. The experimental yield in the transformed extract was FOS $16,1 \%$ quantified by HPLC.

Key words: fructooligosaccharides, FOS, carob, Prosopis pallida, pectinase

\section{INTRODUCCIÓN}

Prosopis pallida (algarrobo) es una leguminosa que crece de manera silvestre en las regiones áridas principalmente del norte peruano desde tiempos ancestrales, produce vainas (algarrobas) de hasta $30 \mathrm{~cm}$ de largo, con elevado contenido de carbohidratos. Las algarrobas contienen: sacarosa $46 \%$, proteínas $8 \%$, grasas $0,7 \%$ y fibra $32 \%{ }^{1}$.

Los carbohidratos tipo fructanos están formados por polímeros de fructosa, unidos mediante enlaces glucosídicos del tipo $\beta$ (2-1), son conocidos como oligofructanos, tienen un grado de polimerización entre tres y nueve unidades de monosacáridos ${ }^{2}$. Los FOS están presentes en diversas especies vegetales como betarraga, alcachofa, entre otros. Asimismo, estos compuestos se producen por hidrólisis enzimática de inulina, levano, entre otros, o por reacciones de síntesis mediante la acción de transferasas e invertasas en un medio rico en sacarosa $^{3}$. Los FOS contienen un residuo terminal de $\alpha$-D-glucosa, y entre ellos destaca: 1-kestosa (GF2), 1-nistosa (GF3) y 1F-fructosilnistosa (GF4) ${ }^{4}$. Este bioproceso genera FOS y glucosa libre, siendo esta última directamente proporcional al proceso de transfructosilación.

En los últimos años, se han descrito enzimas que presentan actividad fructosiltransferasa, tales como invertasas, inulinasas, pectinasas, entre otras. Así, la pectinasa de Aspergillus aculeatus presenta actividades de pectinatranseliminasa, poligalacturonasa, pectinesterasa, $\beta$-galactosidasa, fructosiltransferasa, hemicelulasa y celulasa. Esta enzima, por presentar alta actividad fructosiltransferasa, es utilizada en la producción de FOS a partir de sacarosa. La eficiencia de conversión se mejora mediante la eliminación simultánea de glucosa a través de una reacción enzimática ${ }^{5}$. En este aspecto, la disponibilidad del extracto de algarrobo con alta concentración de carbohidratos motivó la producción de FOS con el propósito de poner en valor a los frutos subutilizados de esta leguminosa. 


\section{PARTE EXPERIMENTAL}

Materiales y reactivos. Las vainas de algarrobo (algarroba) se recolectaron de la provincia de Piura, distrito de Catacaos a $28 \mathrm{~m}$ de altitud. Las algarrobas fueron seleccionadas, pesadas y secadas a $42{ }^{\circ} \mathrm{C}$ hasta peso constante. Luego, las vainas fueron molidas, la harina se cernió con tamices de poros 4 y $1 \mathrm{~mm}$. Por otro lado, los reactivos utilizados fueron: pectinasa de Aspergillus aculeatus, Merck (EE.UU.), glucosa oxidasa, Valtek (Chile) y Fructan Kit, Megazyme (EE.UU.). Los estándares de 1-cestosa, nistosa, sacarosa, glucosa y fructosa, Sigma Chemical (EE.UU). Los otros productos químicos fueron adquiridos de Merck Peruana.

Preparación y análisis del extracto de algarrobo. En un recipiente de acero inoxidable se mezcló la harina de algarroba con agua en una relación de 1:5 (peso/volumen) y se calentó a $80{ }^{\circ} \mathrm{C}$ por $2 \mathrm{~h}$. Después, la mezcla se filtró y el extracto se concentró a $50^{\circ} \mathrm{Brix}$. Al extracto se determinó los sólidos solubles totales (SST) utilizando un refractómetro manual (Giardino, Italia), la actividad de agua a $25^{\circ} \mathrm{C}$ utilizando el método AOAC 978.18. El pH se determinó con un potenciómetro digital (Hanna, Rumania). Los contenidos de azúcares se analizaron usando el método de glucosa oxidasa, DNS y Fructan kit.

Optimización de la producción de FOS. El diseño Box-Behnken se utilizó en la optimización de la producción de FOS usando pectinasa. Para ello, se consideraron cuatro factores: tiempo (h), temperatura $\left({ }^{\circ} \mathrm{C}\right)$, concentraciones de enzima $(\mathrm{mg} / \mathrm{ml})$ y extracto de algarrobo $\left({ }^{\circ} \mathrm{Bx}\right)$. Así, se obtuvieron 27 ensayos y seis puntos centrales que se ejecutaron en tres bloques. Los rangos de los parámetros evaluados fueron de 20 a $50^{\circ} \mathrm{Bx}$ de extracto de algarrobo, pectinasa de 5 a $30 \mu \mathrm{L}$, tiempo de 0,5 a 18 h y temperatura de 45 a $65{ }^{\circ} \mathrm{C}$, siendo variables independientes del proceso. El resultado de 10 de los 54 experimentos se presenta en la tabla 1. Para cada ensayo se mezcló pectinasa y extracto de algarrobo $1 \mathrm{~mL}$. La enzima se inactivó a $100{ }^{\circ} \mathrm{C}$ durante $5 \mathrm{~min}$. Posteriormente, las concentraciones de glucosa, azúcares reductores y FOS se determinaron usando glucosa oxidasa, DNS y Fructan Kit, respectivamente.

La metodología de superficie de respuesta se aplicó al optimizar los datos experimentales utilizando Minitab (versión 16). La ecuación de regresión permitió describir la relación entre la respuesta (FOS y glucosa libre) y los términos incluidos en el modelo, las condiciones óptimas obtenidas por el paquete estadístico fueron ensayadas y los azúcares cuantificados por HPLC. 
Tabla 1. Matriz del diseño Box-Behnken para optimizar la producción de FOS.

\begin{tabular}{ccccc}
\hline $\begin{array}{c}\mathrm{N}^{\circ} \text { de } \\
\text { ensayo }\end{array}$ & Tiempo (h) & Temperatura $\left({ }^{\circ} \mathrm{C}\right)$ & Enzima $(\mu \mathrm{l})$ & $\begin{array}{c}\text { Extracto de } \\
\text { algarrobo }\left({ }^{\circ} \mathrm{B}\right)\end{array}$ \\
\hline 1 & 9,25 & 45 & 17,5 & 20 \\
2 & 18,00 & 55 & 5,0 & 35 \\
3 & 9,25 & 65 & 17,5 & 50 \\
4 & 9,25 & 65 & 17,5 & 20 \\
5 & 18,00 & 55 & 17,5 & 50 \\
6 & 9,25 & 45 & 17,5 & 50 \\
7 & 18,00 & 45 & 17,5 & 35 \\
8 & 18,00 & 45 & 17,5 & 35 \\
9 & 9,25 & 55 & 5,0 & 50 \\
10 & 18,00 & 55 & 17,5 & 50 \\
\hline
\end{tabular}

La significancia estadística al $95 \%$ de confianza, en términos de la ecuación de regresión, se examinó mediante análisis de varianza (ANOVA). Se generaron gráficas de contorno y superficie de respuesta después del análisis por el mismo software.

Cuantificación de carbohidratos en condiciones óptimas de producción de FOS Se realizó por cromatografía líquida de alto rendimiento (HPLC) con un sistema isocrático combinado con detección por índice de refracción para el análisis de carbohidratos. Se aplicaron $20 \mu \mathrm{L}$ de muestra en una columna Aminex HPX 42- (BioRad, Richmond), con un flujo de 0,6 mL/ min, a aproximadamente 52 bars y $81,7^{\circ} \mathrm{C}$; el solvente fue agua mili-Q. Los resultados se analizaron mediante BioCrom, versión 3,0. La interpretación y la cuantificación se realizaron comparando los tiempos de retención y la concentración de las soluciones de carbohidratos estándares. Así, sacarosa, glucosa, fructosa y fructooligosacáridos se prepararon a la concentración de $0,0,5,1,2,4,6,8$ y $10 \mathrm{mg} / \mathrm{ml}$.

\section{RESULTADOS Y DISCUSIÓN}

La harina de los frutos de Prosopis pallida es utilizada en alimentación animal y en menor proporción en humanos, esta harina contiene carbohidratos entre $40 \%$ y $60 \%$, corresponde a fructuosa, glucosa y sacarosa ${ }^{7}$. Además de estos carbohidratos existen galactomananos provenientes del endospermo de las semillas, que presentan unidades de galactosa y manosa con enlaces en posición $\beta-1,4$ que unen varias ramas de D-galactosa mediante enlaces glicosidicos $(1,6)$ cada cuatro o cinco manosas, estos últimos representarían hasta $20 \%$ dependiendo del suelo y la variedad del cultivo5. El análisis proximal de la harina de algarrobo realizado para esta investigación presentó la siguiente composición: carbohidratos $75,5 \%$, proteínas 5,7 \%, grasa $2,1 \%$, cenizas $8,1 \%$; y humedad $8,6 \%$. 
Características físico químicas del extracto de algarrobo. Para obtener el extracto de algarrobo se ensayaron tres concentraciones de harina de algarrobo $10 \%, 20 \%$ y $30 \%$, alcanzando 15, 30 y $40{ }^{\circ} \mathrm{Bx}$, respectivamente, después de ser concentrado a un mismo volumen, lo cual demuestra una mayor recuperación de carbohidratos al $20 \%$ de harina. Finalmente, se preparó el extracto de algarrobo llevándolo hasta una concentración de 50 ${ }^{\circ} \mathrm{Bx}$. El pH y la actividad de agua fueron de 4,5 $\pm 0,01$ y 0,9, respectivamente. Además, el extracto de algarrobo contenía sacarosa $23,82 \%$, glucosa $9,74 \%$ y fructosa $10,53 \%$ y oligosacáridos $4,5 \%$; estos valores comparados con el jugo de caña de azúcar que presenta: fructuosa $2,0 \%$, glucosa $2,0 \%$ y sacarosa $75,0 \%{ }^{6}$ indican que el extracto de algarrobo posee una tercera parte de sacarosa con respecto al de caña de azúcar, concentración importante para su aprovechamiento en la biosíntesis de FOS.

Asimismo, en el extracto de algarrobo se determinaron los siguientes minerales expresados en $\mathrm{mg} / \mathrm{kg}$ : hierro 6, calcio 462, magnesio 372, zinc 6 y fósforo 899 , lo cual indica que este producto es una fuente de carbohidratos y minerales para la alimentación.

Producción enzimática de FOS. En la actualidad, la obtención de FOS se realiza por hidrólisis enzimática de inulina y lévano o por reacciones de síntesis enzimática de hidrólisis y transferencia de fructosas a concentraciones de sacarosa mayores a $100 \mathrm{~g} / \mathrm{l}$, en estas condiciones la invertasa transfiere específicamente moléculas de fructosa a glucosa o sacarosa y forma $\mathrm{FOS}^{3}$. En la biosíntesis de FOS a partir de sacarosa se utilizan diversas enzimas comerciales tales como: inulinasas, invertasas, celulasas, levansacarasas con actividad fructosiltransferasa que catalizan la transferencia de un grupo fructosilo desde una sacarosa a diferentes aceptores, entre ellos, otra molécula de sacarosa, produciendo FOS, estas enzimas pueden estar libres o inmovilizadas, también se utilizan células vivas como Pichia pastoris transgénica, entre otros ${ }^{7}$.

En la producción de FOS a partir de sacarosa se evaluaron diferentes factores y condiciones, así Rubio et al ${ }^{3}$ utilizaron invertasa de Aspergillus niger IB56 y estudiaron el pH a 3,0; 4,0; 4,$5 ; 5,0$ y 5,5 ; temperaturas $20,25,30$ y $40{ }^{\circ} \mathrm{C}$, concentración de sacarosa 15,$0 ; 30,0$ y 45,0 \% y tiempos de incubación a 60; 90 y 120 min. La máxima producción de FOS 10,5 \% la obtuvieron con sacarosa $30,0 \%$; a pH 5,0;20 ${ }^{\circ} \mathrm{C}$ y 60 min de reacción ${ }^{3}$. Por el contrario, PérezCruz et $a l^{7}$ produjeron $32,2 \%$ de FOS, principalmentel-kestosa utilizando Pichia pastoris inmovilizadas en alginato de calcio, en un biorreactor tipo tanque agitado de $5 \mathrm{~L}$. A la vez, Ureta et $\mathrm{al}^{8}$ sintetizaron FOS utilizando sacarosa 20, 30 y $55 \%$ (p/v) y Viscozyme L 3,5 \%, de esta forma obtuvieron FOS $30 \%$ de cadena corta (GF3 y GF4) con sacarosa $30 \%{ }^{8}$. En tanto, Vega et al9 utilizaron preparaciones enzimáticas comerciales, como Pectinex Ultra SP-L con $6 \mathrm{~h}$ de reacción, el rendimiento fue entre 58,8 a 62,6 \% (g sc-FOS/100 g de sacarosa). En este aspecto, el extracto de algarrobo es un sustrato mucho más complejo en comparación a soluciones saturadas de sacarosa. Además, el seguimiento del bioproceso resultaba costoso, por ello se realizaron ensayos preliminares con diversas enzimas comerciales como inulinasa, invertasa y pectinasa con sacarosa al $30 \%$, y se encontraron rendimientos de 12,1 $\%, 11,5 \%$, y 14,0 \% de glucosa, respectivamente. En estos experimentos se evaluaron las concentraciones de glucosa en el medio de reacción. La liberación de esta molécula fue directamente proporcional a la de síntesis de FOS. 
En los últimos años, la demanda de FOS por las industrias alimentaria y farmacéutica se incrementó, por ello se busca convertir insumos alimentarios nativos con altas concentraciones de sacarosa a FOS utilizando procesos limpios y sostenibles como reacciones enzimáticas usando herramientas estadísticas para su optimización. Así, Vega et al ${ }^{10}$ analizaron temperatura, concentraciones de sacarosa y enzima (celulasas Rohapect $\mathrm{CM}$ ) en el medio de reacción para la producción de FOS de cadena corta (sc-FOS). Las respuestas del diseño fueron las siguientes: rendimiento (g sc-FOS/100 g de sacarosa), 1-kestosa (g/100 g sc-FOS) y productividad volumétrica (g sc-FOS / L h). Las condiciones óptimas fueron $50^{\circ} \mathrm{C}, 6,6$ $\mathrm{U} / \mathrm{ml}$ de enzima, sacarosa 2,103 $\mathrm{M}$ en tampón de acetato $50 \mathrm{mM}$ a pH 5,5, y $5 \mathrm{~h}$. En estas condiciones, se lograron sGF2 de $68,2 \%{ }^{10}$. Por este lado, el método de superficie de respuesta con el diseño Box-Behnken disminuye los costos de cuantificación de FOS en el proceso, tanto por el método enzimático como por HPLC (tabla 1), los intermedios de las variables independientes fueron: $9,25 \mathrm{~h}, 55^{\circ} \mathrm{C}$, enzima $35,9 \mathrm{mg}$ y extracto de algarrobo $35^{\circ} \mathrm{Bx}$.

Los porcentajes de FOS, glucosa libre y azúcares reductores de 15 de los 54 ensayos de transfructosilación se presentan en la tabla 2. En la matriz del MSR según el diseño Box-Benhen se observa que los porcentajes de glucosa tanto el menor (1,6\%) como el mayor (10,11\%) están en los ensayos $\mathrm{N}^{\circ} 20$ y 39 , respectivamente, en las condiciones de $9,25 \mathrm{~h}, 55^{\circ} \mathrm{C}, 17,5 \mu \mathrm{L}$ de enzima y $35^{\circ} \mathrm{Bx}$ de extracto de algarrobo. En todos los ensayos, la concentración de glucosa es directamente proporcional a la biosíntesis de fructooligosacáridos en el medio de reacción. Con respecto a la producción de FOS, fue menor en el ensayo $\mathrm{N}^{\circ} 34$ con 2,41 \% y mayor en los ensayos $\mathrm{N}^{\circ} 15,26,31,39$ y 41, en los que se alcanzó en los que se alcanzó una media de $13,81 \%$ de FOS en el medio de reacción.

Tabla 2. Producción de FOS vía enzimática a partir del extracto de algarrobo según el diseño Box-Behnken del MSR.

\begin{tabular}{cccccccc}
\hline $\mathrm{N}^{\circ}$ ensayo & $\mathrm{T}(\mathrm{h})$ & $\mathrm{TR}\left({ }^{\circ} \mathrm{C}\right)$ & $\mathrm{E}(\mu \mathrm{l})$ & $\mathrm{CS}\left({ }^{\circ} \mathrm{Bx}\right)$ & $\mathrm{G}(\%)$ & $\mathrm{AR}(\%)$ & $\mathrm{FOS}(\%)$ \\
\hline 1 & 9,25 & 45 & 17,5 & 20 & 4,66 & 8,94 & 11,01 \\
2 & 18,00 & 55 & 5,0 & 35 & 8,99 & 13,15 & 11,26 \\
3 & 9,25 & 65 & 17,5 & 50 & 6,82 & 17,45 & 11,45 \\
4 & 9,25 & 65 & 17,5 & 20 & 4,29 & 7,87 & 12,75 \\
5 & 18,00 & 55 & 17,5 & 50 & 8,06 & 14,02 & 9,56 \\
6 & 9,25 & 45 & 17,5 & 50 & 5,19 & 17,28 & 12,99 \\
7 & 18,00 & 45 & 17,5 & 35 & 8,33 & 14,87 & 12,98 \\
8 & 18,00 & 45 & 17,5 & 35 & 8,40 & 14,56 & 13,04 \\
9 & 9,25 & 55 & 5,0 & 50 & 8,43 & 15,78 & 12,09 \\
10 & 18,00 & 55 & 17,5 & 50 & 8,55 & 14,11 & 8,70 \\
11 & 9,25 & 45 & 5,0 & 35 & 9,30 & 12,33 & 9,91 \\
12 & 9,25 & 55 & 5,0 & 50 & 8,89 & 15,97 & 12,70 \\
13 & 9,25 & 45 & 30,0 & 35 & 7,19 & 13,49 & 7,07 \\
14 & 9,25 & 45 & 30,0 & 35 & 7,56 & 13,84 & 7,34 \\
15 & 9,25 & 55 & 17,5 & 35 & 9,45 & 16,03 & 13,88 \\
\hline
\end{tabular}

Leyenda: T, tiempo; TR, temperatura de reacción; E, enzima; CS, concentración de sustrato o extracto de algarrobo; FOS, fructooligosacáridos; G, glucosa libre; AR, azúcares reductores libres; ${ }^{\circ} \mathrm{Bx}$, grados Brix. 
Al calcular el coeficiente de regresión de superficie del porcentaje FOS se encontró $\mathrm{R}^{2}$ de $90,36 \%, \mathrm{R}^{2}$ predictivo de $80,8 \%$ y $\mathrm{R}^{2}$ ajustado de $86,89 \%$. En tanto, el porcentaje de glucosa $\mathrm{R} 2$ es $89,44 \%, \mathrm{R}^{2}$ predictivo $79,08 \%$ y $\mathrm{R}^{2}$ ajustado $85,64 \%$ (tablas 3 y 4 ). Los coeficientes de determinación $\mathrm{R}^{2}$ señalan que la síntesis de FOS y glucosa libre se ajustan al modelo empleado. Las interacciones tiempo-enzima y temperatura-enzima en la biosíntesis de FOS presentaron un $\mathrm{p}<0,05$ (tablas 3 y 4), por lo que es estadísticamente significativo.

Tabla 3. Coeficiente de regresión de superficie de respuesta del porcentaje FOS vs. tiempo y temperatura.

\begin{tabular}{|c|c|c|c|c|}
\hline EE del término & Coef. & Coef. & $\mathrm{T}$ & $\mathrm{P}$ \\
\hline Constante & $-55,9573$ & 10,6026 & $-5,278$ & 0,000 \\
\hline Tiempo (h) & 1,3857 & 0,2360 & 5,872 & 0,000 \\
\hline Temperatura $\left({ }^{\circ} \mathrm{C}\right)$ & 1,8737 & 0,3205 & 5,846 & 0,000 \\
\hline Enzima (ul) & 0,2142 & 0,1685 & 1,271 & 0,211 \\
\hline Algarrobo $\left({ }^{\circ} \mathrm{Bx}\right)$ & 0,4960 & 0,1514 & 3,277 & 0,002 \\
\hline Tiempo (h)*Tiempo (h) & $-0,0430$ & 0,0036 & $-11,838$ & 0,000 \\
\hline Temperatura $\left({ }^{\circ} \mathrm{C}\right) *$ Temperatura $\left({ }^{\circ} \mathrm{C}\right)$ & $-0,0184$ & 0,0028 & $-6,605$ & 0,000 \\
\hline Enzima (ul)*Enzima (ul) & $-0,0176$ & 0,0018 & $-9,864$ & 0,000 \\
\hline Algarrobo $\left({ }^{\circ} \mathrm{Bx}\right) *$ Algarrobo $\left({ }^{\circ} \mathrm{Bx}\right)$ & $-0,0075$ & 0,0012 & $-6,098$ & 0,000 \\
\hline Tiempo (h)*Temperatura $\left({ }^{\circ} \mathrm{C}\right)$ & $-0,0007$ & 0,0037 & $-0,195$ & 0,846 \\
\hline Tiempo (h)*Enzima (ul) & $-0,0159$ & 0,0029 & $-5,422$ & 0,000 \\
\hline Tiempo (h)*Algarrobo $\left({ }^{\circ} \mathrm{Bx}\right)$ & $-0,0008$ & 0,0024 & $-0,319$ & 0,751 \\
\hline Temperatura $\left({ }^{\circ} \mathrm{C}\right) *$ Enzima (ul) & 0,0082 & 0,0026 & 3,194 & 0,003 \\
\hline Temperatura $\left({ }^{\circ} \mathrm{C}\right) *$ Algarrobo $\left({ }^{\circ} \mathrm{Bx}\right)$ & $-0,0002$ & 0,0021 & $-0,076$ & 0,940 \\
\hline Enzima (ul)*Algarrobo $\left({ }^{\circ} \mathrm{Bx}\right)$ & 0,0036 & 0,0017 & 2,103 & 0,042 \\
\hline \multicolumn{5}{|l|}{$\mathrm{S}=0.908322 \quad$ PRESS $=64.1191$} \\
\hline R-cuad. $=90,36 \%$ R-cuad. $(\mathrm{p}$ & $8 \%$ & & & \\
\hline
\end{tabular}

La ecuación de segundo orden simula correctamente la síntesis de fructooligosacáridos de acuerdo al diseño Box-Behen.

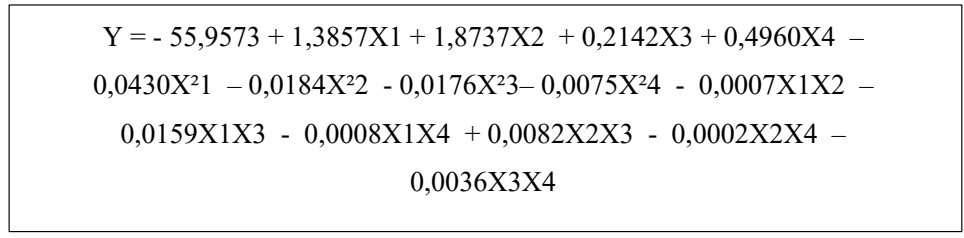

Leyenda: Y, concentración de fructooligosacáridos; X1, tiempo en horas; X2, temperatura ${ }^{\circ} \mathrm{C}$; X3, Concentración de enzima; X4, Concentración de extracto en ${ }^{\circ} \mathrm{Bx}$. 


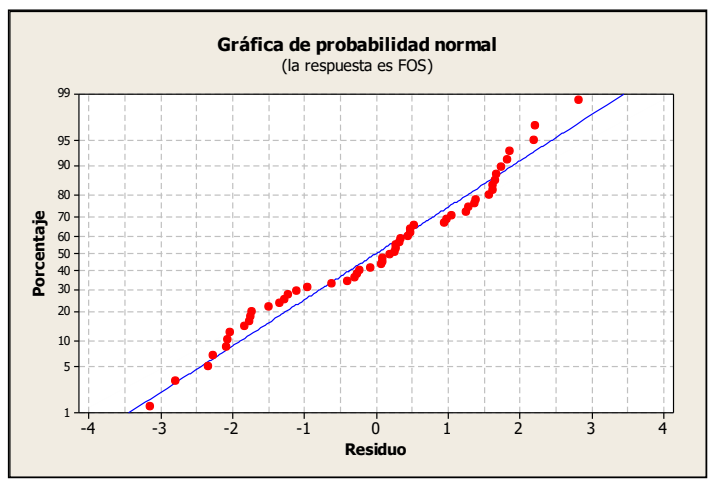

Figura 1. Gráfica de distribución normal de residuos del porcentaje de FOS.

Análisis gráfico de la producción de FOS. Los gráficos de superficie de respuesta y contorno (figuras 2 y 3 ) fueron establecidos entre dos factores, mientras los demás se mantuvieron en un nivel intermedio. Asimismo, la liberación de glucosa al medio fue directamente proporcional a la biosíntesis de FOS. Este resultado permitió monitorear el proceso de biosíntesis de FOS de forma indirecta usando un método enzimático fácil, rápido y económico.

El paquete estadístico Minitab 16 fue utilizado para generar gráficas de superficie y contorno usando las variables que influyen en el proceso de transfructosilación como tiempo (h), temperatura $\left({ }^{\circ} \mathrm{C}\right)$, enzima $(\mu \mathrm{L})$ y extracto de algarrobo $\left({ }^{\circ} \mathrm{Bx}\right)$ muestran los valores óptimos. Así, en la figura 2a se observa que el porcentaje más bajo de FOS (Z) se obtiene cuando la concentración de extracto de algarrobo (Y) es $20^{\circ} \mathrm{Bx}$ y enzima (X) $30 \mu \mathrm{l}$ y el más alto a intervalos de 30 a $40{ }^{\circ} \mathrm{Bx}$ y 15 a $20 \mu \mathrm{l}$. Paralelamente, se observa en la figura $2 \mathrm{~b}$ que el porcentaje más alto de glucosa se obtiene en un intervalo de tiempo y temperatura de 10 a 15 h y 54 a $60{ }^{\circ} \mathrm{C}$, respectivamente.
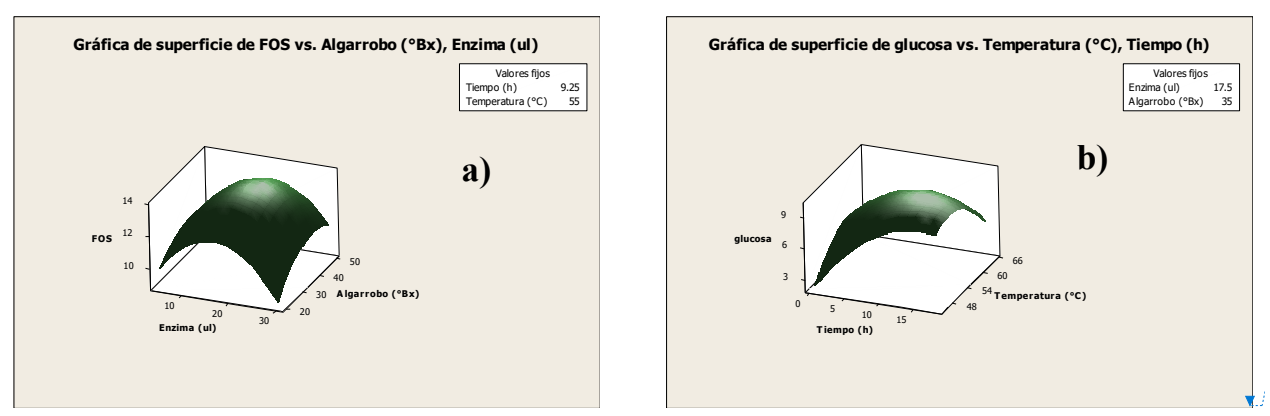

Figura 2. Gráficas de superficie de respuesta de los factores que afectan la producción de FOS a partir del extracto de algarrobo: a) porcentaje de FOS versus enzima y extracto de algarrobo; b) porcentaje de glucosa producida versus tiempo y temperatura. 
La figura 3 muestra cómo el tiempo (T), la temperatura (TP), la concentración de enzima (CE) y el extracto de algarrobo (EA) afectan las reacciones de transfructosilación en la biosíntesis de FOS (Z). Las regiones más oscuras indican los valores con mayor producción de FOS, los niveles de contorno revelan un pico central en $12 \mathrm{~h}, 54{ }^{\circ} \mathrm{C}, 17.5 \mu \mathrm{l}$ de enzima y $54{ }^{\circ} \mathrm{Bx}$ de extracto de algarrobo. En estas condiciones la producción de FOS (Z) fue $\geq 13 \%$.

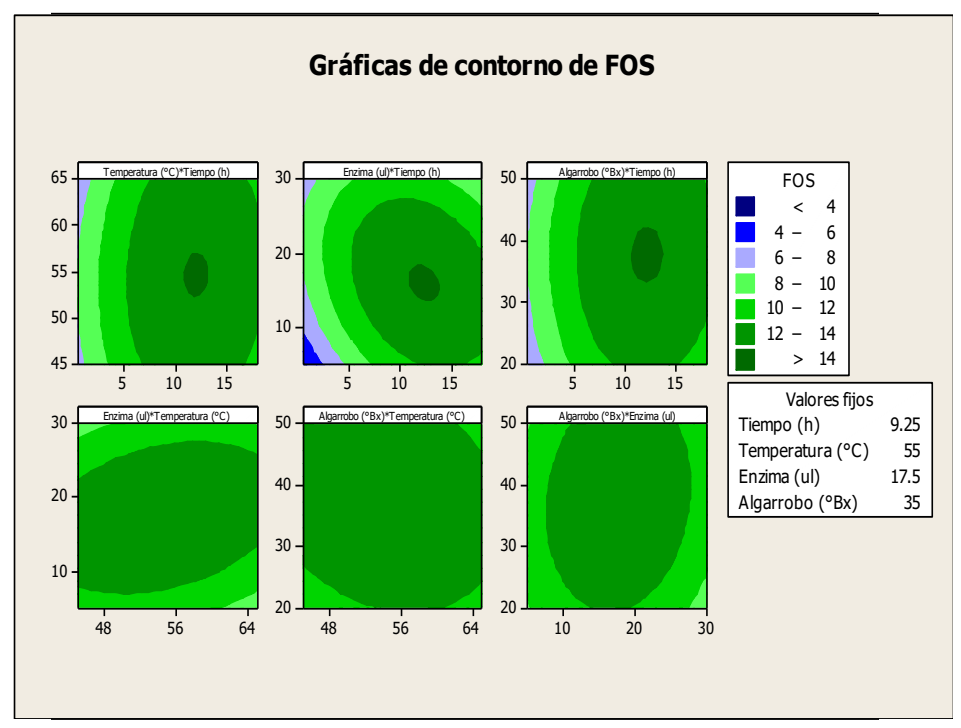

Figura 3. Gráficas de contornos del porcentaje de la producción de FOS versus tiempo, temperatura, enzima y extracto de algarrobo. a) temperatura y tiempo, b) enzima y tiempo, c) extracto y tiempo, d) enzima y temperatura, e) extracto y temperatura, f) extracto y enzima.

También se realizó la cuantificación de glucosa en el medio de reacción considerando la tabla matriz del diseño Box-Behnken, ya que la liberación de glucosa es concomitante tanto en las reacciones de hidrólisis como transfructosilación. 
Tabla 4. Coeficiente de regresión de superficie de respuesta del porcentaje de glucosa vs tiempo y temperatura.

\begin{tabular}{|c|c|c|c|c|}
\hline EE del término & Coef. & Coef. & $\mathrm{T}$ & $\mathrm{P}$ \\
\hline Constante & $-81,2627$ & 10,2620 & $-7,919$ & 0,000 \\
\hline Tiempo (h) & 2,0408 & 0,2284 & 8,935 & 0,000 \\
\hline Temperatura $\left({ }^{\circ} \mathrm{C}\right)$ & 2,2975 & 0,3102 & 7,406 & 0,000 \\
\hline Enzima (ul) & 0,3007 & 0,1631 & 1,844 & 0,073 \\
\hline Algarrobo $\left({ }^{\circ} \mathrm{Bx}\right)$ & 0,8144 & 0,1465 & 5,558 & 0,000 \\
\hline Tiempo (h)*Tiempo (h) & $-0,0286$ & 0,0035 & $-8,136$ & 0,000 \\
\hline Temperatura $\left({ }^{\circ} \mathrm{C}\right) *$ Temperatura $\left({ }^{\circ} \mathrm{C}\right)$ & $-0,0207$ & 0,0027 & $-7,675$ & 0,000 \\
\hline Enzima (ul)*Enzima (ul) & $-0,0018$ & 0,0017 & $-1,030$ & 0,309 \\
\hline Algarrobo $\left({ }^{\circ} \mathrm{Bx}\right)^{*}$ Algarrobo $\left({ }^{\circ} \mathrm{Bx}\right)$ & $-0,0098$ & 0,0012 & $-8,206$ & 0,000 \\
\hline Tiempo (h)*Temperatura $\left({ }^{\circ} \mathrm{C}\right)$ & $-0,0176$ & 0,0036 & $-4,957$ & 0,000 \\
\hline Tiempo (h)*Enzima (ul) & $-0,0114$ & 0,0028 & $-4,023$ & 0,000 \\
\hline Tiempo (h)*Algarrobo $\left({ }^{\circ} \mathrm{Bx}\right)$ & $-0,0040$ & 0,0024 & $-1,696$ & 0,098 \\
\hline Temperatura $\left({ }^{\circ} \mathrm{C}\right) *$ Enzima $(\mathrm{ul})$ & 0,0023 & 0,0025 & 0,911 & 0,368 \\
\hline Temperatura $\left({ }^{\circ} \mathrm{C}\right) *$ Algarrobo $\left({ }^{\circ} \mathrm{Bx}\right)$ & 0,0018 & 0,0021 & 0,874 & 0,378 \\
\hline Enzima $(\mathrm{ul}) *$ Algarrobo $\left({ }^{\circ} \mathrm{Bx}\right)$ & $-0,0070$ & 0,0017 & $-4,213$ & 0,000 \\
\hline$S=0.879148 \quad$ PRESS $=59.6796$ & & & & \\
\hline
\end{tabular}

El valor expresado por el coeficiente de determinación $\mathrm{R}^{2}$ indicó que el 89,44 \% de variabilidad en la respuesta se explica por el diseño propuesto.

Optimización de la respuesta. De acuerdo al análisis de respuesta del porcentaje de FOS, se determinaron los parámetros de optimización que se presentan en la figura 5 con el fin de maximizar las reacciones de transfructosilación, así como obtener el mayor porcentaje de FOS en el extracto de algarrobo.

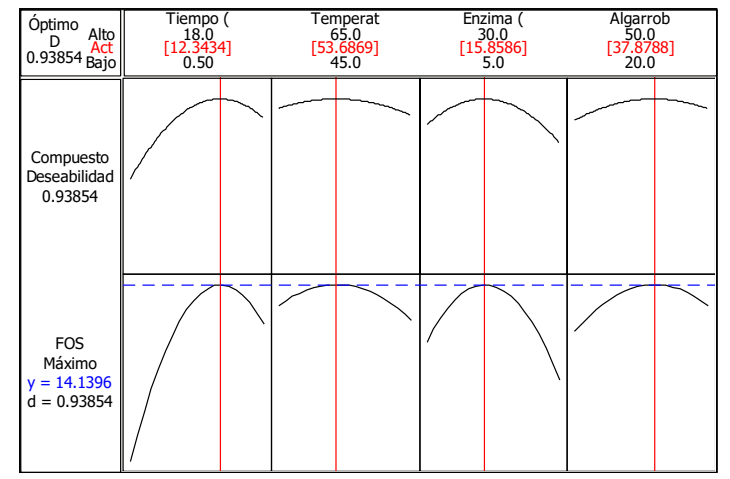

Figura 4. Gráfica que indica las condiciones óptimas para el proceso de transfructosilación. 
Validación del modelo para la producción de FOS. De acuerdo a los análisis y ajustes realizados se determinaron que las condiciones óptimas de producción de FOS a partir de azúcares simples son: $12 \mathrm{~h} ; 54^{\circ} \mathrm{C}$; enzima $16 \mu \mathrm{l}(31 \mathrm{mg})$ y extracto de algarrobo $38^{\circ} \mathrm{Bx}$, para un rendimiento teórico de $14 \%$, siendo el valor experimental 16,10\%. En estas condiciones se cuantificaron los carbohidratos presentes en el medio de reacción por HPLC (figura 4, tabla 5). En las muestras MP-EA0 (extracto de algarrobo crudo) y MP- EAD (extracto de algarrobo digerido) se obtuvieron 4,5 y 17,30 g de oligosacáridos totales, respectivamente, equivalentes a 16,10 g FOS por $100 \mathrm{~g}$ de extracto de algarrobo (tabla 5), siendo éste mayor a los reportados por Surín et al ${ }^{11}$ quienes obtuvieron 14,36 \% de FOS (nistosa (GF3) 2,03 \% y kestosa (GF2) 12,33 \%) a partir del jarabe de Logan. En tanto, Muñoz ${ }^{6}$, utilizando jugo de caña y células permeabilizadas de Candida apícola, obtuvo FOS 4,5\%., ${ }^{6,11}$.

Tabla 5. Determinación de carbohidratos en los extractos de algarrobo mediante HPLC.

\begin{tabular}{cccccc}
\hline Muestra & $\begin{array}{c}\text { Fructosa* } \\
*(\mathrm{~g} / 100 \mathrm{~g})\end{array}$ & $\begin{array}{c}\text { Glucosa* } \\
(\%)\end{array}$ & $\begin{array}{c}\text { Sacarosa* } \\
(\%)\end{array}$ & $(\%)$ & \multicolumn{2}{c}{ Oligosacáridos $(\%)$} \\
\cline { 5 - 6 } MP-EAO & 8,00 & 7,40 & 18,10 & 0,00 & FOS* \\
MP-EAD & 5,80 & 12,6 & 2,30 & 16,10 & 1,20 \\
\hline
\end{tabular}

Leyenda: Donde: MP-EAO, Extracto de algarrobo inicial, MP-EAD; Extracto de algarrobo digerido.

En el cromatograma (figura 5) se muestran los tiempos de retención de FOS biosintetizado. Se observa la presencia de sc-FOS como kestosa (GF2), nistosa (GF3) y fructosilnistosa (GF4), con un mayor pico en GF2, lo cual sugiere la mayor concentración de este fructósido, similar a lo obtenido por Vega et $a l^{10}$ con sacarosa y Surin et al ${ }^{11}$ con el jarabe de Logan ${ }^{10,1}$.

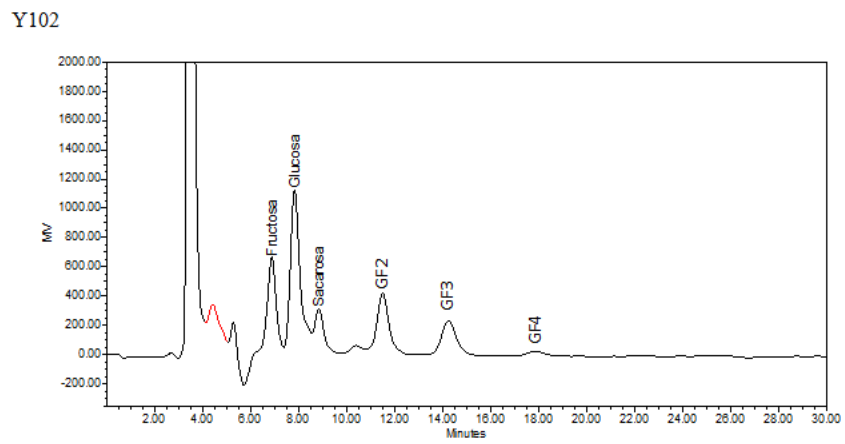

Figura 5. Perfiles cromatográficos obtenidos por HPLC del extracto digerido de algarrobo. 
La cuantificación de los diferentes polímeros de fructooligosacáridos producidos a partir del extracto de algarrobo por síntesis enzimática permitirá comparar mejor los resultados con los descritos por otros investigadores. Sin embargo, las condiciones óptimas de biosíntesis de FOS obtenidas en este estudio servirán para la prospección a nivel industrial y la valorización de un cultivo subutilizado para su uso como ingrediente alimentario saludable.

\section{CONCLUSIONES}

Las condiciones óptimas para la producción de FOS $16.1 \%$ a partir del extracto de algarrobo $38^{\circ} \mathrm{Bx}$ fueron Pectinex Ultra SP-L 31,6 mg a $54^{\circ} \mathrm{C}$ y $12 \mathrm{~h} 20 \mathrm{~min}$. De este modo, el extracto de algarrobo se convierte en un ingrediente alimentario saludable alternativo elaborado con tecnología limpia y sostenible.

\section{AGRADECIMIENTO}

Este proyecto ha sido financiado por el VRI - UNMSM.

\section{REFERENCIAS BIBLIOGRÁFICAS}

1. Tamayo L, Arteaga D y Jaramillo J. Propiedades farmacológicas del Algarrobo (Hymenaea courbaril Linneaus) de interés para la industria de alimentos. Rev. Lasallista Investig. 2008; 5 (2):100-111

2. Flores-Maltos DA, Mussatto SI, Contreras-Esquivel JC, Rodríguez-Herrera R, Teixeira JA, Aguilar CN. Biotechnological production and application of fructooligosaccharides. Crit Rev Biotechnol. 2016;36(2):259-267.

3. Rubio C, Latina A, Antonio N. Producción de fructooligosacaridos por invertasa de Aspergillus niger Ib56: un prebiótico de importancia para la salud humana y animal. Bol Micol. 2012; 27: 18-23.

4. Pérez-Conesa D, López G, Ros G. Principales prebióticos y sus efectos en la alimentación. An. Vet. (Murcia). 2004; 20: 5-20

5. Sciammaro L, Ferrero C, Puppo C. Agregado de valor al fruto de Prosopis alba. Estudio de la composición química y nutricional para su aplicación en bocaditos dulces saludables. Rev Fac Agron. 2015; 114 (1): 115-123.

6. Muñoz Equihua E. Escalamiento del proceso de obtención de fructooligosacáridos a partir de jugo de caña mediante síntesis enzimática con células permeabilizadas de Candida apicola [Tesis de Maestría]. Zapopan, Jalisco (México): Centro de Investigación y Asistencia en Tecnología y diseño del Estado de Jalisco, A. C.; 2016.

7. Pérez-Cruz E, Martínez-García D, Sobrino-Legón A, Rodriguez-Rico A, Hernández L. Escalado de la reacción de biosíntesis de fructooligosacáridos, a partir de sacarosa, en biorreactores tipo tanque agitado. RTQ. 2011; 31(2): 19-25 
8. Ureta MM, Romano N, Kakisu E, Gómez-Zavaglia A. Synthesis of fructooligosaccharides using grape must and sucrose as raw materials. Food Res Int. 2019; 123:166-171.

9. Vega-Paulino RJ, Zúñiga-Hansen ME. Potential application of commercial enzyme preparations for industrial production of short-chain fructooligosacharides. J Mol Catal B Enzym. 2012; 76: 44-51.

10. Vega R. Zúniga-Hansen ME. Enzymatic synthesis of fructooligosaccharides with high 1-kestose concentrations using response surface methodology. Bioresour Technol. 2011; 102(22):10180-10186.

11. Surin S, Seesuriyachan P, Thakeow P, Phimolsiripol Y. Optimization of enzymatic production of fructooligosaccharides from longan syrup. J Appl Sci. 2012; 12: 11181123 